\title{
Aplicación de nuevas técnicas en la caracterización de humedales relacionados con el agua subterránea
}

\author{
Leandro Rodrigues Capítulo(1), Patricia Laurencena ${ }^{(2)}$, Juan Manuel García(2), Nora Gómez ${ }^{(1)}$ y \\ Eduardo Emilio Kruse(1)
}

(1) Consejo Nacional de Investigaciones Científicas y Técnicas (CONICET). CEIDE - UNLP. Calle $64 n^{\circ}$ 3. La Plata. Buenos Aires. Argentina. leandrorodriguescapitulo@gmail.com; nora@ilpla.edu.ar; kruse@fcnym.unlp.edu.ar

(2) Comisión de Investigaciones Científicas (CIC). CEIDE - UNLP, calle $64 n^{\circ} 3$. La Plata. Buenos Aires. Argentina. plauren@fcnym.unlp.edu.ar; jm.garcia689@gmail.com

\begin{abstract}
RESUMEN
La aparición de herramientas tecnológicas modernas facilita la caracterización, monitoreo y modelados de humedales relacionados con el agua subterránea. Estas técnicas incluyen el uso de sistemas de aeronaves no tripuladas (drones) y sensores de medición continua de los niveles y calidad del agua. Los procedimientos tradicionales asociados a estas nuevas tecnologías brindan una oportunidad para avanzar con mayor precisión en el conocimiento hidrológico de los humedales. Se presentan los principales lineamientos de la metodología empleada en un estudio que abarca humedales situados en el noreste de la Provincia de Buenos Aires. Se realizaron relevamientos fotogramétricos con un dron tipo multirotor equipado con un sensor RGB de alta resolución. Las configuraciones de vuelo, altura, velocidad, solapamiento frontal y lateral y grilla de recorrido fueron programadas para evaluar las variaciones areales de los humedales. Se realizó el procesado de la información mediante softwares específicos. A partir de relevamientos de campo se ejecutaron perforaciones de monitoreo en las proximidades del humedal en las cuales se instalaron sensores para la medición continua de los niveles, conductividad eléctrica del agua y temperatura. Los resultados a obtener consisten en mosaicos ortorectificados de alta resolución, modelos digitales de elevación y modelos 3D del terreno, que muestran las variaciones areales del agua en superficie del humedal. Ello asociado a los datos registrados en las perforaciones, permite un análisis espacio - temporal a distintas escalas de la relación agua superficial agua subterránea en el humedal.
\end{abstract}

Palabras clave: agua subterránea, drones, humedales, técnicas de estudio.

\section{Application of new techniques in the characterization of wetlands related to groundwater}

\begin{abstract}
The appearance of modern technological tools facilitates the characterization, monitoring, and modelling of wetlands related to groundwater. These techniques include the use of unmanned aircraft systems (drones) and continuous measurement sensors for water levels and quality. The traditional procedures associated with these new technologies provide an opportunity to advance the hydrological knowledge of wetlands with greater precision. The main guidelines of the methodology used in a study covering wetlands located in the northeast of the Province of Buenos Aires are presented. Photogrammetric surveys were carried out with a multi-rotor type drone equipped with a high-resolution RGB sensor. The configurations of flight, height, speed, frontal and lateral overlap and route grid were programmed to evaluate the areal variations of the wetlands. The information was processed using specific software. From field surveys, monitoring drilling was carried out in the vicinity of the wetland, where sensors were installed for the continuous measurement of levels, electrical conductivity of water and temperature. The results obtained consist of high-resolution orthorectified mosaics, digital elevation models and $3 D$ terrain models, which show the areal variations of water in
\end{abstract}


the wetland surface. This, associated with the data recorded in the drilling, allows a spatiotemporal analysis at different scales of the surface water - groundwater relationship in the wetland.

Keywords: drone, groundwater, study techniques, wetland.

\section{Introducción}

Desde que en 2007 aparecieron los drones aéreos o vehículos aéreos no tripulados (UAV) con cámaras fotográficas y costos asequibles al público en general, se ha generalizado su uso como herramienta en geología e hidrología.

En la actualidad se puede desarrollar cartografía temática de diversos ámbitos en el campo de la hidrogeología gracias a la resolución espacial de los sensores instalados en drones, que varía desde milímetros hasta metros.

La introducción de importantes innovaciones de este tipo de equipamiento, así como los softwares asociados para fotogrametría han ampliado de manera significativa sus prestaciones en la exploración aérea. La aeronave no tripulada permite tiempos de vuelo reducidos y llegar a sectores de difícil acceso; resultando en una disminución importante del tiempo y costos de adquisición de datos y de toda la operación de monitoreo (Capolupo et al. 2015; Nex y Remondino, 2014).

Los humedales son zonas donde el agua es el prin- cipal factor controlador del medio y la vida vegetal y animal asociada a él (Ramsar, 2006). Consisten en rasgos de la superficie de laTierra cuya existencia responde a la combinación de numerosos factores y que se manifiestan mediante la existencia de agua estancada en forma somera o que fluye muy lentamente (Custodio, 2001). En general su aparición viene acompañada por la existencia de una superficie freática a escasa profundidad, la cual posibilita el desarrollo y permanencia de vegetación freatofita aún durante períodos de sequía prolongada (Manzano, 2005).

En los humedales del noreste de la Provincia de Buenos Aires se reconocen bañados asociados a planicies de inundación. Se trata de ambientes con agua en superficie de manera permanente o semipermanente, de contorno o perímetro indefinido y con vegetación emergente abundante. Este tipo de humedales ofrece múltiples beneficios entre ellos se destaca su función de actuar como filtros naturales capaces de mejorar la calidad de agua que circula aguas abajo. También es reconocido que estos cuerpos de agua pueden reducir los picos de inundación mediante el almacenamiento temporal del agua, retardando su

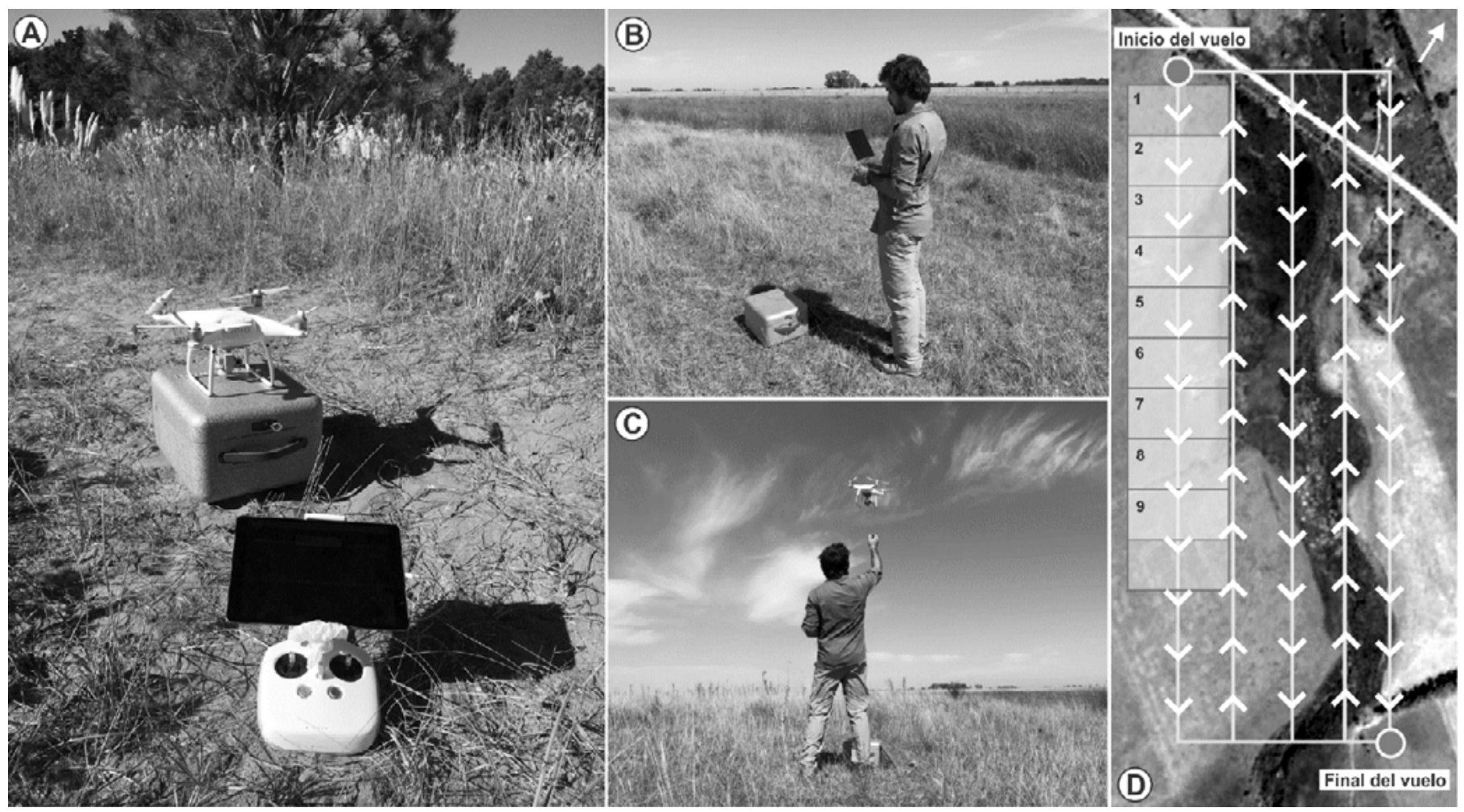

Figura 1. Relevamiento fotogramétrico. Preparación del equipo y geoposicionamiento (A), monitoreo del vuelo (B), final del vuelo (C) y grilla de vuelo (D).

Figure 1. Photogrammetric survey. Preparation of equipment and geolocation (A), flight monitoring $(B)$, end of flight (C) and flight grid (D). 
efecto aguas abajo. Asimismo, son lugares ricos en biodiversidad, sirviendo de refugio para muchas especies (Rodrigues Capitulo et al., 2017).

Se presentan los principales lineamientos de la metodología empleada, con especial referencia al uso de drones en humedales situados en el noreste de la Provincia de Buenos Aires, así como también algunos ejemplos de su utilización. Este trabajo se relaciona con el proyecto financiado por la ANPCYT PICT 20151342.

\section{Metodología}

La caracterización de los humedales en el área de estudio se llevó a cabo a partir de vuelos aerofotogramétricos realizados mediante el empleo un dron multirotor Phantom IV Pro equipado con un sensor RGB de alta definición, sensor infrarrojo para detección de obstáculos, sistema estabilizador automático en vuelo, altura máxima de $500 \mathrm{msnt}$ (metros sobre el nivel del terreno) y una velocidad límite de $15 \mathrm{~m} / \mathrm{s}$ (Figuras $1 \mathrm{~A}, 1 \mathrm{~B}$ y $1 \mathrm{C}$ ).

Este equipo también cuenta con un control remoto de doble frecuencia el cual le otorga una distancia máxima de vuelo de $8 \mathrm{~km}$ respecto de la aeronave, así como también brinda en simultáneo las imágenes y videos capturados por el dron.
Para la configuración del vuelo se utiliza la aplicación Pix4D V3.2.0 la cual permite seleccionar la mejor relación de solape de fotogramas, altura de vuelo, velocidad de vuelo, grado de detalle del pixel, etc. Esta tarea se realiza en gabinete con conexión a internet debido a que es necesario la descarga de imágenes y datos en línea los cuales son exportados posteriormente al equipo de dron.

Es importante considerar la altitud de las estructuras presentes que pueden obstaculizar el recorrido del dron durante el relevamiento. Para los humedales en el área de estudio, dichos obstáculos se relacionan con la vegetación arbórea (álamos, fresnos y eucaliptus).

Para la elección de la grilla de vuelo en los casos estudiados se contemplaron las bajas pendientes del terreno (menores al 1\%) que caracterizan a la llanura pampeana. Con relación al solapamiento y con el objeto de garantizar la fiabilidad tanto de los ortomosaicos como también del modelo digital de elevación, se seleccionó el distanciamiento óptimo entre transectas de vuelo permitiendo un solapamiento mayor al $60 \%$ (Figura 1 D). La velocidad de vuelo se fijó con el plan de permitir el relevamiento de toda el área mediante el empleo de una única batería.

La configuración seleccionada permitió el relevamiento de distintos humedales. Para un relevamien-
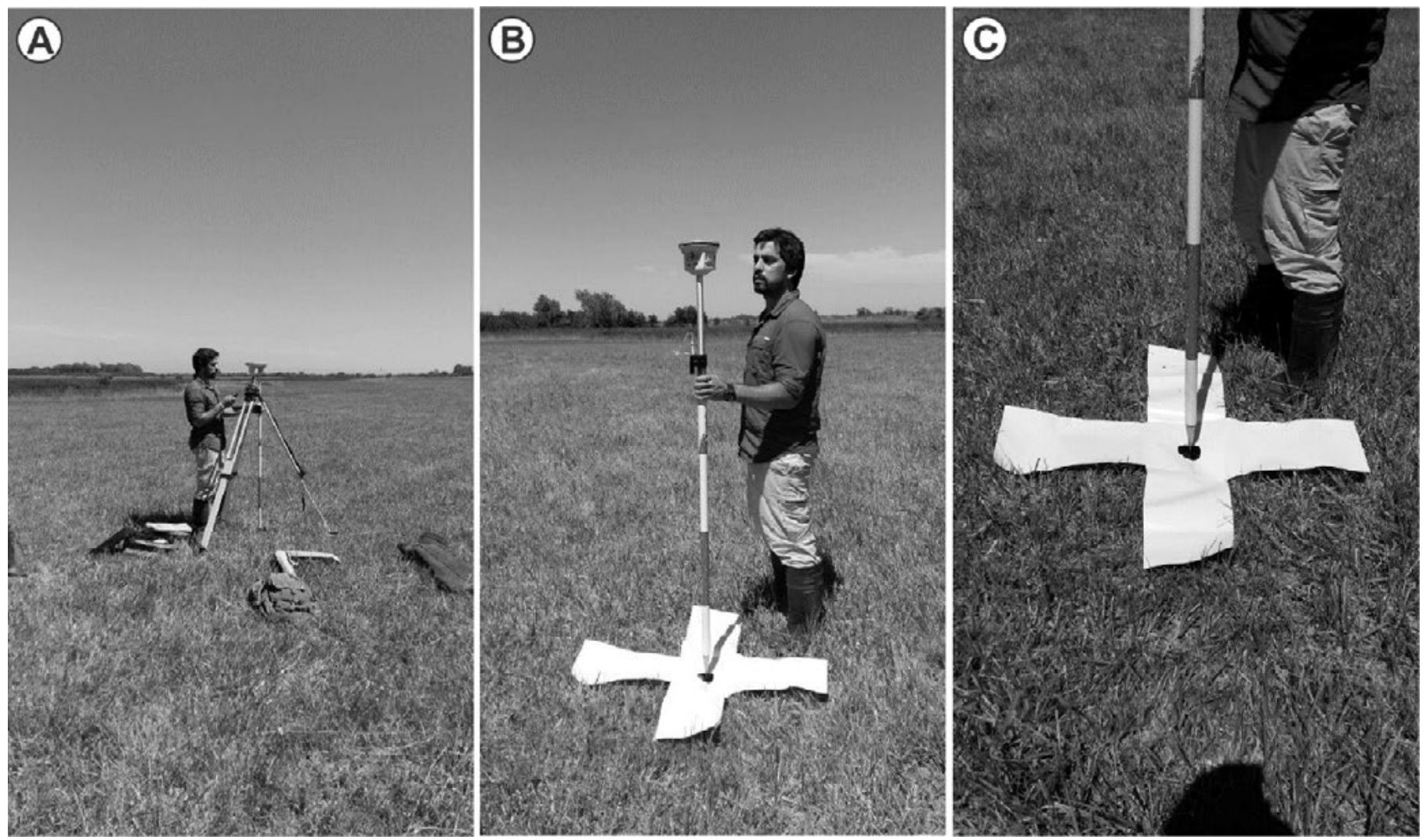

Figura 2. Acotamiento de los marcadores topográficos con equipo de GPS diferencial. Figure 2. Height measurement of topographic markers with differential GPS equipment. 
to del orden de 30 hectáreas se emplearon grillas de $1000 \mathrm{~m}$ de longitud por $300 \mathrm{~m}$ de ancho. La tarea se realizó a una altura de vuelo de $120 \mathrm{msnt}$, en menos de 15 minutos, obteniéndose aproximadamente 300 fotogramas incluidas en 6 o 7 transectas.

La verificación de los valores planialtimétricos obtenidos con el dron requieren el acotamiento de puntos de control situados en sectores estratégicos (Figura 2). Los puntos acotados, con una precisión de centímetros, sirven como red de apoyo para validar los datos obtenidos por el dron durante el procesamiento en gabinete.

En el procesamiento de la información obtenida por el dron se emplearon softwares específicos. Se confeccionó un modelo digital de elevación (DEM) y un ortomosaico georreferenciado y ortorectificado que integra todas las tomas realizadas resultando en una imagen de detalle con una resolución espacial del orden $3 \mathrm{~cm} /$ pixel.

Para la transformación del DEM a MDT (modelo digital del terreno) en primer lugar se realizó la clasificación de los puntos del terreno. Este proceso permite discriminar aquellos pixeles asociados a la vegetación de aquellos que resultan del terreno propiamente dicho. Posteriormente se creó una malla en donde los puntos asociados a la vegetación fueron excluidos permitiendo obtener mapas de curvas topográficas acordes al terreno estudio.

Para la integración de la información generada se emplea un sistema de información geográfica, a partir del cual fue posible la creación de diferentes capas raster y vectoriales consistentes en mapas de dirección y acumulación de flujo; delimitación de cuencas e identificación de la red drenaje principal y secundaria cuyos productos permitieron el análisis con diferentes escalas de detalle.

\section{Resultados}

Se presentan a modo de ejemplo los relevamientos efectuados con un dron en dos sitios en marzo y junio de 2018. Se ubican en las nacientes de los Arroyos Cajaravillas y Chubichamini (Figura 3). Se trata de un ámbito Ilano de escasa pendiente, en donde el acuí-



Figura 3. Ubicación del área de estudio.

Figure 3. Location of the study area. 


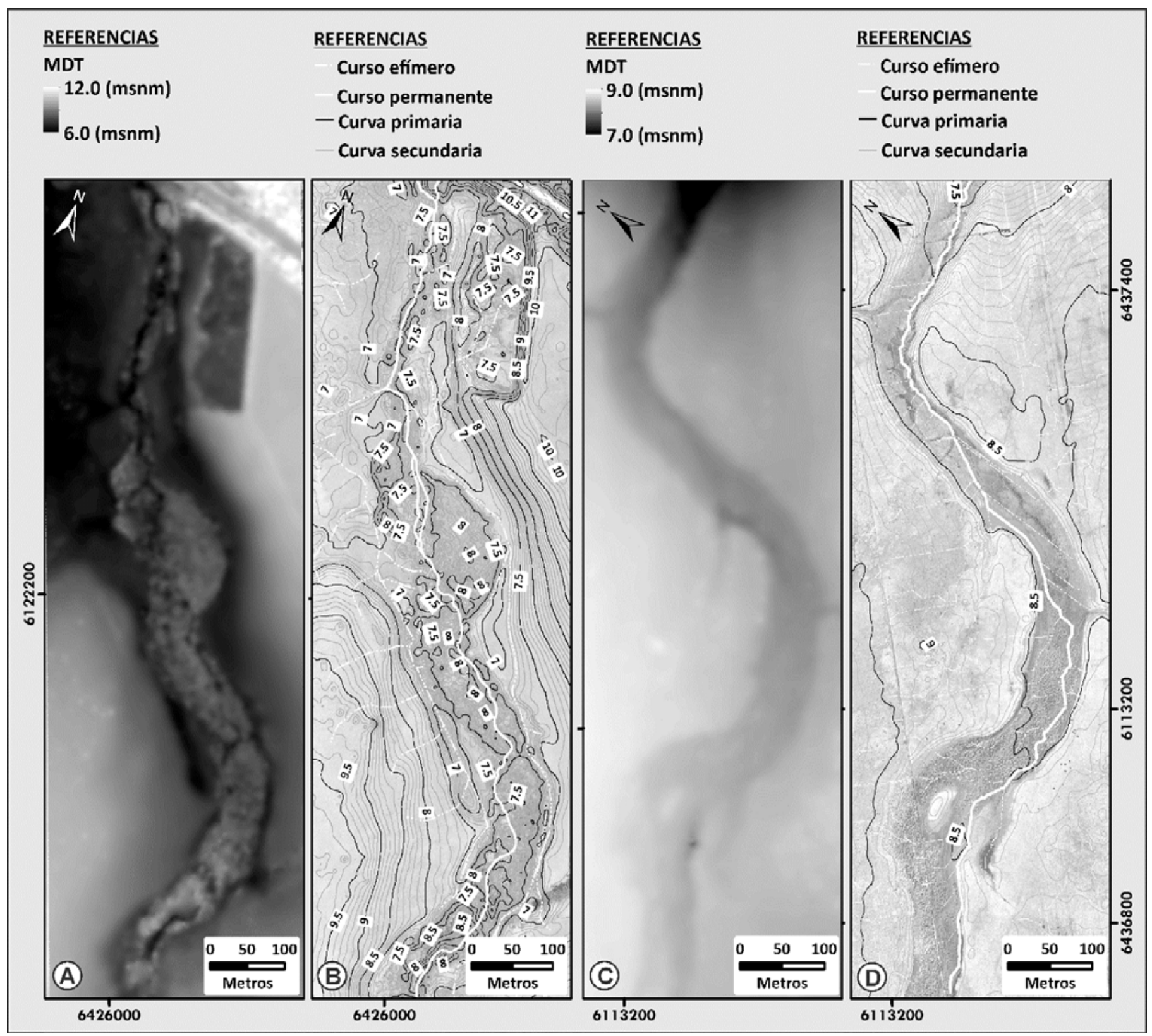

Figura 4. Modelo digital del terreno, mapa topográfico y red de drenaje del Arroyo Cajaravillas (A y B). Modelo digital del terreno, mapa topográfico y red de drenaje del Arroyo Chubichamini (C y D).

Figure 4. Digital terrain model, topographic map and drainage network of the Cajaravillas Stream (A and B). Digital terrain model, topographic map and drainage network of Chubichamini Stream (C and D).

fero freático, de escasa profundidad (entre 1 y $3 \mathrm{~m}$ ) presenta un escurrimiento local hacia los cursos y regionalmente al Río de la Plata.

El procesado de los fotogramas posibilitó la creación de un MDT a partir del cual se realizaron los mapas topográficos y la red de drenaje en cada caso (Figura 4).

Los mapas generados permitieron identificar con detalle las particularidades hidrológicas del humedal. Se reconoce una red de drenaje dendrítica compleja cuyo curso principal presenta un gradiente de $3.5 \times 10^{-4}$ en el caso del Chubichamini y de $1.2 \times 10^{-3}$ en el Cajaravillas.

El grado de detalle alcanzado en la confección de la red de drenaje de los cursos efímeros resulta de interés hidrológico ya que permite reconocer el movimiento del agua superficial en situaciones de precipitaciones extremas y su relación con los diferentes grados de humedad asociada al afloramiento de la capa freática.

Esta tarea permitió no sólo el reconocimiento e identificación de los cursos permanentes y efímeros, 
sino también las variaciones areales de los cuerpos de agua, el contenido de humedad en el suelo y el estado de la vegetación para dos estados hidrológicos. Todo ello favorece la compresión del funcionamiento hidrológico del sistema. Debe tenerse en cuenta que, en la dinámica de este sistema de llanura, donde predominan la evapotranspiración e infiltración, el escurrimiento superficial se encuentra condicionado por
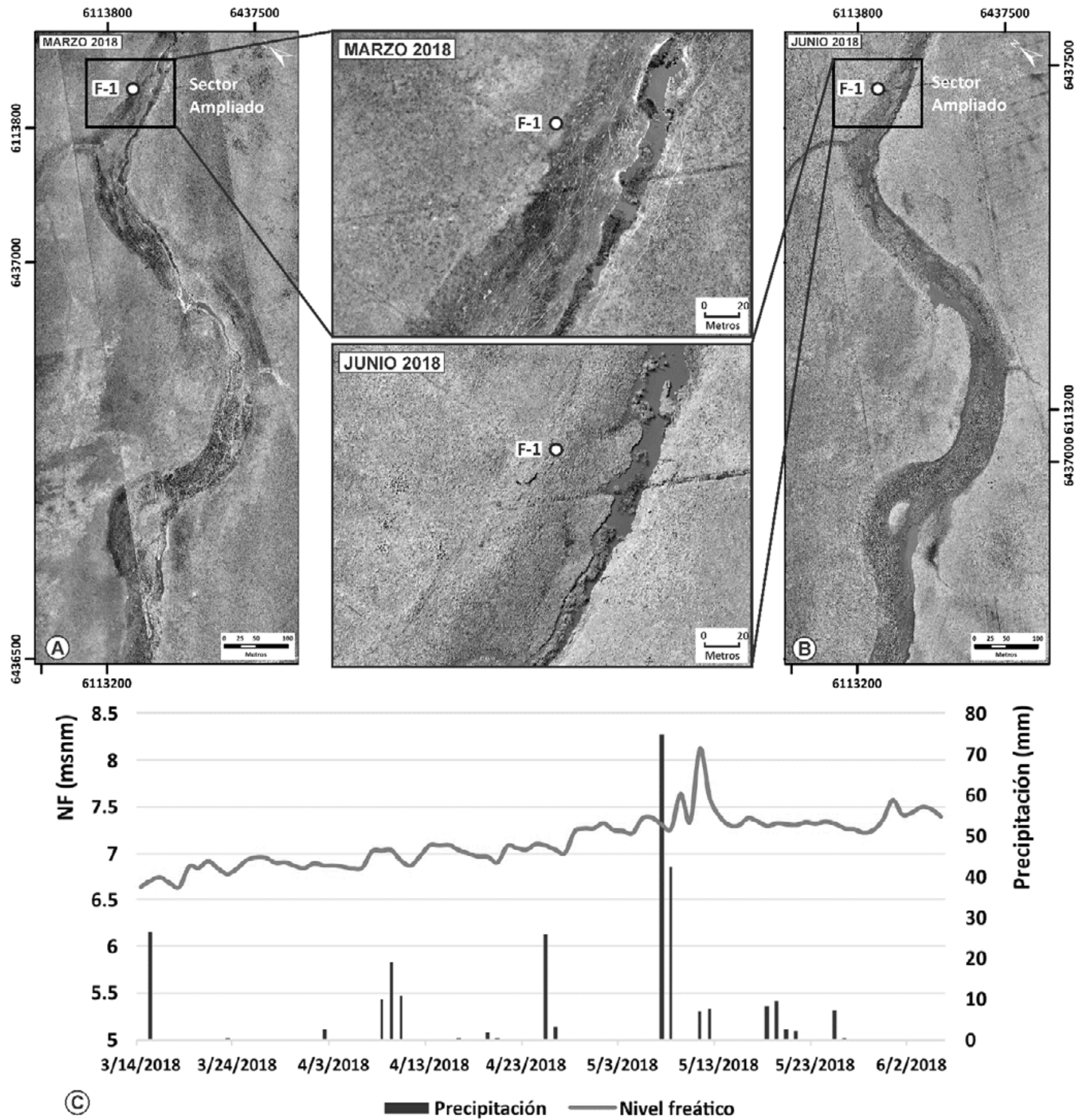

Figura 5. Ortomosaicos del sector de humedal del Arroyo Chubichamini para los meses de marzo (A) y junio (B) de 2018. En C, se muestra la evolución del nivel freático registrado por el diver de nivel en el sondeo F-1 (Círculo).

Figure 5. Orthomosaics of the wetland sector of the Chubichamini Stream for the months of March (A) and June (B) 2018. In C, the evolution of the phreatic level recorded by the level diver in the F-1 borehole is shown (circle). 

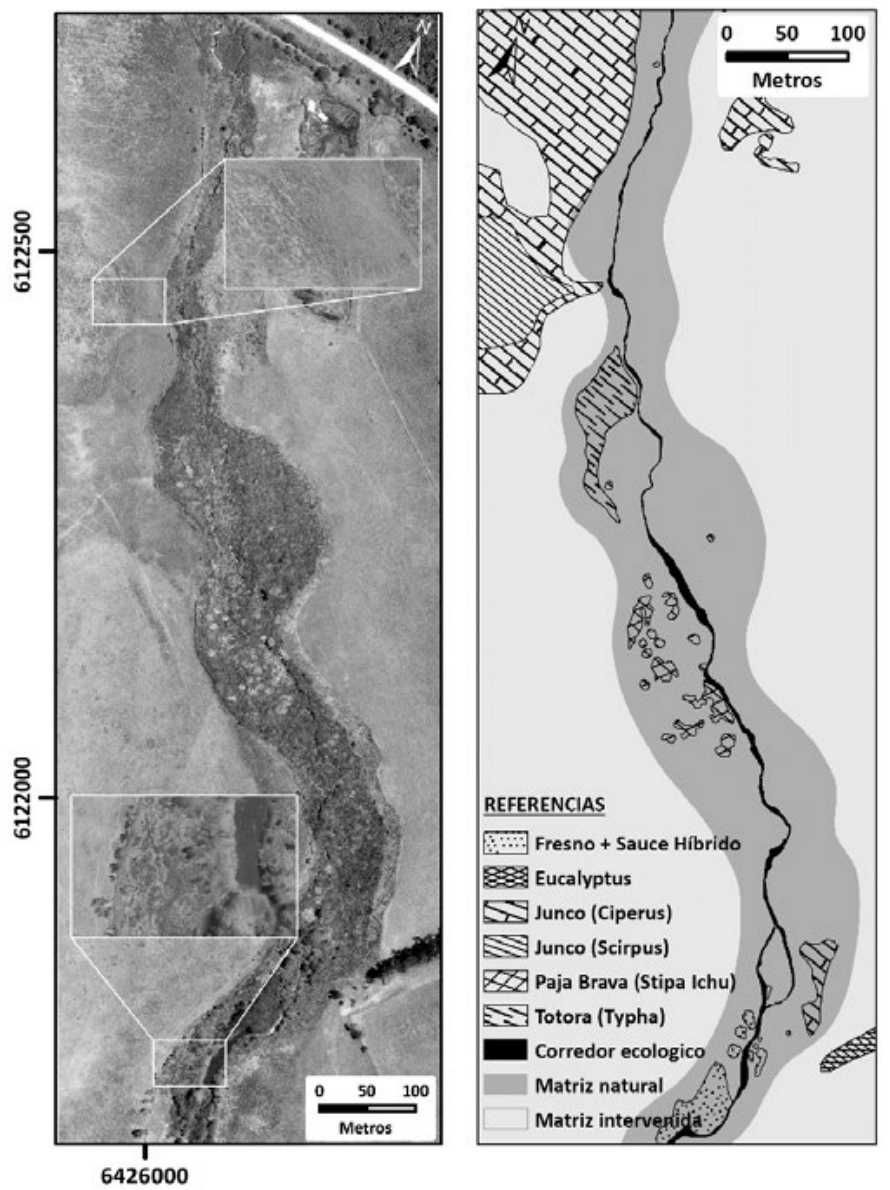

Figura 6. Mapa temático de especies herbáceas y arbóreas del Arroyo Cajaravillas.

Figure 6. Thematic map of herbaceous and arboreal species of the Cajaravillas Stream.

diferencias de alturas menores a 10 o $20 \mathrm{~cm}$.

También ello tiene significado limnológico, en particular en aquellos procesos vinculados con la remoción y transporte de nutrientes en superficie (fósforo, nitrógeno, materia orgánica, sedimentos, etc.) luego de eventos de precipitación y que son los responsables del crecimiento y desarrollo de las especies vegetales en cada caso.

En la Figura 5, se muestran dos situaciones hidrológicas del Arroyo Chubichamini, correspondientes a los días 14 de marzo y 2 junio de 2018 (Figuras $5 \mathrm{~A}$ y 5B) y la evolución de los niveles freáticos para un sondeo de monitoreo ubicado próximo al curso de agua. En el lapso existente entre los dos relevamientos se produjo una disminución en el espesor de la zona no saturada de aproximadamente $1,5 \mathrm{~m}$. Se pueden reconocer sectores con agua en superficie y sectores con diferentes contenidos de humedad. El detalle de las fotos permite diferenciar los ámbitos que presentan acumulación de agua en superficie, las variaciones producidas entre una y otra época, las áreas del humedal que se relacionan con las variaciones del nivel freático y las variacio- nes del tipo y estadio de la vegetación.

En el Arroyo Cajaravillas, el detalle de las tomas conjuntamente con la verificación de campo permitió la clasificación de la vegetación, la identificación de los diferentes estadios de crecimiento, cuyos distintos contenidos de humedad del suelo y la presencia de la capa freática a escasa profundidad condicionan su manifestación en el terreno (Figura 6). De este modo, se cuenta no sólo con un mapa temático que sirve de mapa base para el análisis ecohidrológico, sino que se puede verificar la influencia de distintos factores hidrológicos en el comportamiento del humedal.

\section{Conclusiones}

La metodología desarrollada a partir del uso de drones aéreos en humedales relacionados con el agua subterránea en ámbitos llanos adquiere significación para la identificación de su estructura y funcionamiento.

El desarrollo de este tipo herramienta facilita la caracterización, monitoreo y modelado de estos humedales. Las principales ventajas se vinculan a:

- Versatilidad, ya que posibilita realizar relevamientos en fechas y horarios asociados a eventos puntuales de interés por su influencia natural o antrópica, con rapidez y a bajo costo.

- Capacidad de análisis en sectores de difícil acceso, lo cual permite la identificación del comportamiento en eventos extremos, ya que se trata de fenómenos extensos y prolongados de anegamiento e inundación, en que los caminos no pueden ser transitados para efectuar otros tipos de relevamiento.

- Elevada resolución espacial, lo cual, en un ambiente de llanura en períodos de estiaje, posibilita asociar las variaciones del agua en superficie o de la humedad en el suelo con las variaciones de los niveles freáticos, todo ello asociado a cambios en la vegetación en el humedal.

- Producción de información hidrológica, morfológica, topográfica, de uso de suelo georeferenciada de interés para el manejo de los recursos naturales y en particular de los recursos hídricos.

\section{Referencias}

Capolupo, A., Pindozzi, S., Okello, C., Boccia, L. 2015. Indirect field technology for detecting areas object of illegal spills harmful to human health: application of drones, photogrammetry and hydrological models. Geospatial Health, 8 (3), 699-707.

Custodio, E. 2001. Aguas subterráneas y humedales. VII Simposio de Hidrogeología. Asociación Española de Hidrología Subterránea. Murcia. 3: 3-27.

Manzano, M. 2005. Hidrología de los humedales dependientes de aguas subterráneas e implicaciones ecológicas. II Seminario Hispano-Latinoamericano sobre Temas Actuales de Hidrología Subterránea: relación aguas superficiales-aguas subterráneas. 
Mónica Blarasín, Adriana Cabrera y Edel Matteoda (eds.). Río Cuarto. (1) 269-290.

Nex, F., Remondino, F., 2014. UAV for 3D mapping applications: a review. Applied Geomatics. 6 (1), 1-15.

Ramsar. 2006. Manual de la convención de Ramsar: Guía a la convención sobre los humedales (Ramsar, Irán, 1971). 4ta edición. Gland. Suiza. Secretaria de la Convención de Ramsar, Gland (Suiza). 124 pp.

Rodrigues Capítulo L., García J.M., Jensen R., Romañuk G.A., Marchesic C., Kruse E., Gómez N. 2017. Técnicas de caracterización hidrogeológica como base conceptual en estudios limnológicos. IX Congreso de Ecología y Manejo de Ecosistemas Acuáticos Pampeanos. La Plata. (36) 124-125.

Recibido: julio 2019

Revisado: noviembre 2019

Aceptado: enero 2020

Publicado: marzo 2021 